\section{New money flows to CARB-X for antimicrobials}

The UK government and the Bill \& Melinda Gates Foundation in May joined a partnership dedicated to the development of new antibiotics, vaccines, diagnostics and other products against drug-resistant bacterial infections. The UK's Global AMR (antimicrobial resistance) Innovation Fund is committing up to $£ 20$ (\$26.6) million and the Bill \& Melinda Gates Foundation up to $\$ 25$ million over the next three years to CARB-X, a non-profit, public-private partnership set up with the mission of averting the threat of drug-resistant diseases. With the new support, CARB-X has now amassed more than $\$ 500$ million to invest through 2021 to accelerate innovation by supporting early-stage projects and phase 1 clinical trials. Geneva-based CARB-X was formed in 2016 with support from UK charity Wellcome Trust, the US Department of Health and Human Services Biomedical Advanced Research and Development Authority (BARDA) and the National Institute of Allergy and Infectious Diseases (NIAID). It currently has 33 projects ongoing in seven countries, including five drug candidates advanced to phase 1 trials and two diagnostics now in the system-integration and testing phase. Of the 27 current drug projects, 11 focus on new targets and 9 on new antibiotic classes. Four are biologics: one is a vaccine against Staphylococcus aureus and the other three, against Pseudomonas aeruginosa, include recombinant lysin protein, a multispecific antibody and an antibody-drug conjugate. Two are small molecules targeting the microbiome: Clostridium difficile and carbapenem-resistant Enterobacteriaceae/vancomycin-resistant Enterococci. Of the six device and/or diagnostic projects, three focus on development of hospital-based systems for identifying bloodstream infections. May 2018 also marked the official launch of the Global AMR Research \& Development Collaboration Hub, announced at the $71^{\text {st }}$ World Health Assembly in Geneva. The initiative was conceived in 2017 with initial support from the Bill \& Melinda Gates Foundation and the Wellcome Trust. The secretariat of the Global AMR R\&D Hub will initially be based in Berlin, at the German Center for Infection Research. Its 18 members also include Russia, China, the US, France and the European Commission.

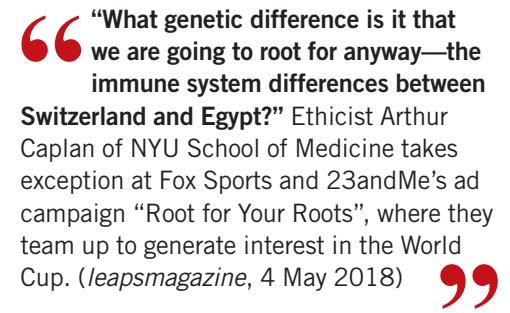

who worked on the early stages of the rice's development and is now executive secretary of the Golden Rice Humanitarian Board, set up to provide strategic guidance to the Golden Rice Project. "That's more mortality than is associated with tuberculosis, malaria or HIV," he says.

But the development of Golden Rice, the first crop engineered with traits to benefit consumers rather than enhance production, has taken far longer than anyone anticipated says Dubock. "I originally thought in 2001 that it would be available by 2003," he says. "Instead it has taken almost 20 years."

In 2001, the Swiss agricultural biotech Syngenta, where Dubock worked, took on Golden Rice development, in a complicated licensing arrangement, involving 23 agreements with 16 licensees, aimed at ensuring the rice would be distributed free to subsistence farmers in the developing world. The company's scientists made several improvements to the rice, such as substituting the daffodil phytoene synthase with an equivalent gene from maize that improved beta-carotene production. The new strain produced up to $37 \mu \mathrm{g}$ per gram, such that a single serving could deliver more than half the recommended daily intake of beta carotene. In 2004, Syngenta withdrew from the project and since then, development has been carried out at independent research institutes, such as the non-profit research and educational institute IRRI, supported by charities including the Rockefeller Foundation and the Bill and Melinda Gates Foundation.

The rice has overcome a number of technical hurdles and controversies along the way. A 2012 study in China, which found that Golden Rice was as effective as beta-carotene oil at providing vitamin A to children, was retracted in 2015 due to a failure to obtain informed consent from the children's parents and faked ethics approval documents.

And in 2017, a study in India that crossed Golden Rice with a local variety found that the resulting plants were stunted and pale, and yield was reduced by as much as $60-70 \%$. Many opponents of genetically modified crops leapt on that result as proof that the project was doomed. But, in general, when researchers insert genes into a plant genome, they must ensure it is done in a way that allows their expression without interfering with other genes. Ashok Singh, a plant geneticist at the Indian Agricultural Research Institute in New Delhi who led the study, identified the problem.

The stunted rice came from a genetic transformation known as the $\mathrm{R}$ event, in which the insertion disrupted an important native membrane transport gene called OsAux1. A different transformation event, called the E event, did not disrupt any native genes and has similar agronomic characteristics to native rice. "The problem had nothing to do with Golden Rice itself, it was event-associated," he says. It was data from rice based on the $\mathrm{E}$ event that were submitted to the FDA and other regulators.

Most scientists expect technical difficulties, says Russell Reinke who leads the healthier rice program at IRRI. "That's just the process of science happening as it should," he says. Singh is concerned, however, about the low carotenoid content of the E-event rice (just $11 \mu \mathrm{g}$ per gram of grain), and its stability in the grain during processing and storage.

The FDA also noted that the levels of beta carotene were too low to make any claims about nutrient content. At such concentrations, the rice is not a viable solution for vitamin deficiency, says Paul Johnston, head of the environmental charity Greenpeace's science unit at the University of Exeter, and puts paid to any nutritional claims. "Given that that's what it is intended to solve, you've got to question what the point of it is," he says.

Greenpeace and other opponents of GM crops say that other interventions, such as increasing consumption of conventionally bred sweet potatoes rich in beta carotene, and ensuring people eat a diverse diet, are more effective at combating vitamin A deficiency than Golden Rice.

But IRRI's Reinke says the FDA's nutritional value calculation was based on the relatively small amount of rice people eat in the US. Based on consumption levels in Asia, where rice makes up to $70 \%$ of the daily calorie intake, Golden Rice could provide as much as half the average daily requirement of vitamin $\mathrm{A}$, he says. Johnston is not sure Golden Rice will ever make it to the field. "It's always in the future, the time span never seems to compress," he says.

But the rice's proponents say they are getting closer to full deployment. The most important next step is receiving regulatory approval from Bangladesh and the Philippines to cultivate and allow people to eat the rice. IRRI submitted applications to both countries last year. Once approved, the Golden Rice trait will still need to be bred into local varieties, and feeding trials will then be necessary to ensure it has the desired nutritional result. Singh estimates it will take at least five to six years after approval to finally get the rice onto people's plates.

Even if regulators give a green light, changing political or social attitudes could add further twists and turns into Golden Rice's long road to acceptance. "If it was just the regulations, I would say it will be very soon. But the politics are very hard to control," says Dubock. "But it is clear that we are getting closer." Brian Owens St. Stephen, New Brunswick, Canada 